\title{
Espectro de gotas e idade de trifólios na taxa de absorção e efeito residual de fungicidas em soja
}

\author{
Drops spectra and leaflets age on the fungicides absorption rate and residual effect in soybean
}

\author{
Giuvan Lenz $^{{ }^{*}}$ Ricardo Silveiro Balardin ${ }^{\mathrm{I}}$ Simone Gripa Minuzzi ${ }^{\mathrm{I}}$ Nédio Rodrigo Tormen ${ }^{\mathrm{I}}$ \\ Leandro Nascimento Marques ${ }^{I}$
}

\section{RESUMO}

O objetivo do presente trabalho foi avaliar o efeito de espectros de gotas (grossas, médias e finas) na velocidade de absorção de fungicidas, para trifólios de diferentes idades através de medida indireta expressa pelo residual de controle de ferrugem asiática da soja (Phakopsora pachyrhizi). O delineamento experimental utilizado foi inteiramente casualizado, com quatro repetições, em arranjo fatorial (3x3x5x4), cujos fatores foram compostos por: três categorias de gotas (grossas, médias e finas); três diferentes posições de avaliação na planta (1ํ. 3o e 5o trifólios); quatro períodos de tempo entre a aplicação de fungicidas e aplicação de chuva simulada (0, 30, 60 e 120min), mais uma testemunha sem chuva; testemunha sem aplicação, mais os fungicidas azoxistrobina + ciproconazol $\left(60+24 g\right.$ i.a. $\left.h a^{-1}\right)+$ óleo mineral $0,6 L \mathrm{ha}^{-1}$, azoxistrobina $\left(50 \mathrm{~g}\right.$ i.a. $\left.h \mathrm{a}^{-1}\right)+$ óleo mineral $0,6 L \mathrm{ha}^{-1}$ e ciproconazol (30g i.a. ha $\left.\mathrm{h}^{-1}\right)$. Avaliou-se a densidade de gotas por centímetro quadrado, diâmetro mediano volumétrico, diâmetro mediano numérico e amplitude relativa, além do número de dias para o aparecimento da primeira pústula. Verificou-se que gotas de menor DMV proporcionam maior velocidade de absorção de fungicidas. Trifólios mais novos absorvem os fungicidas mais rapidamente. A utilização de azoxistrobina + ciproconazol + óleo mineral proporcionou o maior número de dias até o aparecimento da primeira pústula.

Palavras-chave: Phakopsora pachyrhizi, tecnologia de aplicação, estrobilurina, triazol, chuva simulada.

\section{ABSTRACT}

This research aimed to evaluate the effect of large, medium and fine droplets spectra and its interaction with the fungicide absorption rate and leaflets age through indirect measurement expressed by the residual control of Asian soybean rust (Phakopsora pachyrhizi). The experimental design was completely randomized with four replications in a factorial

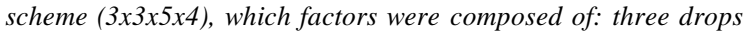
spectra (large, medium and fine); three different positions in the plant evaluated $\left(1^{\text {st }}, 3^{\text {rd }}\right.$ and $5^{\text {th }}$ leaflets $)$, four time periods between the fungicide application and simulated rain $(0,30$, 60 and 120') and a control with no rain; control without fungicide application, Azoxystrobin + Cyproconazole $(60+24 \mathrm{~g}$ a.i. $\left.h \mathrm{a}^{-1}\right)+0.6 \mathrm{~L} \mathrm{ha^{-1 }}$, Azoxystrobin $\left(50 \mathrm{~g}\right.$ a.i. $\left.\mathrm{ha}^{-1}\right)+0.6 \mathrm{~L} \mathrm{ha^{-1 }}$ and Cyproconazole (30g a.i. ha $\left.\mathrm{h}^{-1}\right)$. It was evaluated the density of droplets per square centimeter, volume median diameter, number median diameter and relative amplitude, and the number of days for the first pustule display. It was found that drops with smaller volume median diameter have higher fungicides absorption rates. Newer leaflets provide more quickly fungicides absorption. The use of Azoxystrobin + Cyproconazole provided the greatest number of days until the appearance of the first pustule.

Key words: Phakopsora pachyrhizi, spray technology, strobilurin, triazole, simulated rain, residual.

\section{INTRODUÇÃO}

A ferrugem asiática, causada pelo fungo Phakopsora pachyrhizi Sidow, constitui-se na principal doença da cultura da soja, já que as condições climáticas são favoráveis ao patógeno. Na maioria das regiões, sua alta virulência e velocidade de dispersão são fatores que tornam essa doença altamente agressiva (DEBONA et al., 2008). No que tange aos tecidos das plantas, sabe-se que, com o desenvolvimento da parte aérea, ao mesmo tempo, são

IDepartamento de Defesa Fitossanitária, Centro de Ciências Rurais (CCR), Universidade Federal de Santa Maria (UFSM), 97105900, Santa Maria, RS, Brasil. E-mail: giuvanlenz@gmail.com. *Autor para correspondência. 
encontrados trifólios de diferentes idades que em sua estrutura apresentam diferenças morfológicas e fisiológicas peculiares a cada local. Assim, as características de um fungicida para penetrar nos tecidos foliares são fatores determinantes dentro de uma série de variáveis que determinam a efetividade de um produto (OLIVEIRA et al., 2002).

Poucos são os trabalhos na literatura que relacionam a idade fisiológica dos tecidos com parâmetros relacionados à infecção de Phakopsora pachyrhizi e à absorção de fungicidas. FURTADO et al. (2009), trabalhando com dois cultivares de soja e trifólios de diferentes idades, com o objetivo de avaliar a influência do estádio fenológico e da idade da folha de soja na infecção de $\boldsymbol{P}$. pachyrhizi, observaram que os trifólios mais velhos apresentaram maiores quantidades de doença.

Com a evolução do ciclo, em uma mesma planta, folhas com diferentes idades apresentam distintas características específicas quanto à fisiologia e morfologia, podendo conferir diferentes respostas quanto à absorção de fungicidas.

O objetivo do presente trabalho foi avaliar o efeito de três categorias de gotas (grossas, médias e finas) na velocidade de absorção de fungicidas para trifólios de diferentes idades, através de medida indireta expressa pelo controle de P. pachyrhizi na cultura da soja.

\section{MATERIAL E MÉTODOS}

O experimento foi executado primeiramente em janeiro e repetido em março de 2010 . O delineamento experimental utilizado foi o inteiramente casualizado, com quatro repetições, em arranjo fatorial $(3 \times 3 \times 5 \times 4)$, cujos fatores foram compostos por: três categorias de gotas (grossa, média e fina), avaliações em diferentes posições da planta (1으, 3o e $5^{\circ}$ o trifólios, contados de baixo para cima); quatro períodos de tempo entre a aplicação dos fungicidas e aplicação de chuva simulada $(0,30,60$ e 120min), mais uma testemunha sem chuva, uma testemunha sem aplicação de fungicidas e os produtos azoxistrobina + ciproconazol $\left(60+24 \mathrm{~g}\right.$ i.a. ha $\left.^{-1}\right)$ + óleo mineral Nimbus ${ }^{\circledR} 0,6 \mathrm{~L} \mathrm{ha}^{-1}$, azoxistrobina (50g i.a. ha $\left.^{-1}\right)+$ óleo mineral Nimbus ${ }^{\circledast} 0,6$ ha $^{-1}$ e ciproconazol $\left(30 \mathrm{~g}\right.$ i.a. ha $\left.\mathrm{a}^{-1}\right)$. As doses dos produtos foram definidas conforme recomendações do fabricante.

Os experimentos foram conduzidos em casa de vegetação, sendo a temperatura regulada através de exaustores e a umidade relativa do ar mantida por um sistema de nebulização computadorizado. Para a semeadura, foram utilizadas sementes de soja da cultivar Msoy 8000 RR. Foram semeadas seis sementes por vaso e após a emergência foram mantidas duas plantas de soja por vaso, para aplicação dos tratamentos.

As aplicações de fungicidas foram realizadas quando as plantas atingiram o estádio V5 (FEHR, 1971), em caráter preventivo, utilizando-se pulverizador costal dotado de quatro pontas de pulverização, pressurizado com $\mathrm{CO}_{2}$ e calibrado para uma taxa de aplicação de $150 \mathrm{~L} \mathrm{ha}^{-1}$.

Os diferentes espectros de gotas foram obtidos utilizando-se pontas de jatos planos das séries XR110015, XR11002 eXR11005 para obtenção de gotas das categorias fina, média e grossa, respectivamente, variando a pressão e velocidade durante as aplicações, porém mantendo-se sempre a mesma taxa de aplicação $\left(150 \mathrm{~L} \mathrm{ha}^{-1}\right)$, conforme recomendação do fabricante das pontas (Teejet). Em cada uma das repetições dos tratamentos, foram dispostas estacas divididas em três níveis, correspondentes ao primeiro, terceiro e quinto trifólios das plantas. Para cada nível, foi colocado um papel hidrossensível fixado horizontalmente em placas de metal com um atilho de borracha.

A partir dos cartões coletados, foram obtidas imagens digitais através de scanner, com resolução de varredura de 1200dpi. As imagens foram submetidas à análise computacional pelo software $\mathrm{CIR}^{\circledR}$, versão 1.5 , para determinação da densidade de gotas (DG), diâmetro mediano volumétrico (DMV), diâmetro mediano numérico $(\mathrm{DMN})$ e amplitude relativa (AR) nos três níveis descritos anteriormente.

Após a aplicação dos tratamentos, conforme programado para cada tratamento, foi simulada chuva, utilizando um simulador desenvolvido e validado por DEBORTOLI (2008), trabalhando com intensidade de $255 \mathrm{~mm} \mathrm{~h}^{-1}$ durante quatro minutos. Logo após a simulação da chuva, os vasos foram conduzidos até a casa de vegetação, onde foram mantidos até o final dos experimentos.

Doze horas após a simulação da chuva, os tratamentos foram submetidos à inoculação de $\boldsymbol{P}$. pachyrhizi com uma solução contendo água, espalhante adesivo (Tween 80-100ppm) e uredósporos de $\boldsymbol{P}$. pachyrhizi na concentração de $2 \times 10^{5}$ esporos $\mathrm{mL}^{-1}$. Decorridas 12 horas da inoculação artificial, ao amanhecer, foram iniciadas nebulizações, programadas através de um controlador eletrônico para execução de turno de nebulização de 10 min hora $^{-1}$, mantendo-se assim condições favoráveis ao desenvolvimento do fungo.

Decorridos dois dias da inoculação das plantas, foram feitas avaliações diárias com auxílio de lupa para visualização do aparecimento da primeira pústula esporulada. Nas avaliações, foram considerados o primeiro, terceiro e quinto trifólios de cada planta. 
Os dados obtidos a partir dos dois experimentos conduzidos, foram submetidos inicialmente à análise conjunta, utilizando-se, para tanto, o pacote estatístico SOC ${ }^{\circledR}$ versão 2.1. Como a análise conjunta não mostrou diferenças de resposta dos tratamentos nas duas épocas avaliadas, foi efetuada análise de variância a partir da média das duas épocas.

Os resultados obtidos foram submetidos à análise de variância e a interação entre os fatores, bem como suas médias, foram comparadas através do teste de Tukey a $5 \%$ de probabilidade de erro, com a utilização do pacote estatístico Assistat ${ }^{\circledR}$ versão 7.5 beta (SILVA \&AZEVEDO, 2002).

\section{RESULTADOS E DISCUSSÃO}

A análise de variância das variáveis relacionadas com a deposição de gotas não mostrou interação para nenhum dos fatores analisados, sendo somente significativo ao nível de $1 \%$ de significância o efeito principal dessas variáveis, com exceção da amplitude relativa que apresentou variância não significativa, mesmo para o seu efeito principal.

Considerando as variáveis de deposição de gotas analisadas a partir dos cartões hidrossensíveis, verificou-se que a configuração técnica de operação a que foram submetidas às pontas de pulverização proporcionou um diâmetro mediano volumétrico (DMV) que se enquadra na classificação de padrões de gotas para pulverização, segundo o designado pelo BCPC
(British Crop Production Council), no que foi proposto pelo presente trabalho (Tabela 1). Segundo o BCPC, o DMV para gotas finas corresponde ao intervalo de 119 a $216 \mu \mathrm{m}$, para gotas médias 217 a $352 \mu \mathrm{m}$ e para gotas grossas 354 a $464 \mu \mathrm{m}$.

A análise do DMV mostrou que as três categorias de gotas utilizadas foram diferentes entre si e inversamente proporcionais ao número de gotas por centímetro quadrado (NGC), que também se mostrou diferente para as três categorias. Para o diâmetro mediano numérico (DMN), não foram observadas diferenças entre gotas médias e grossas. Quando a variável avaliada foi à amplitude relativa, fator este que representa a uniformidade das gotas pulverizadas, não foram observadas diferenças entre as categorias, sendo que os valores encontrados de 0,99; 0,97 e 0,98, para espectros de gotas finas, médias e grossas, respectivamente, são compatíveis com o padrão de pulverização determinado pelo fabricante para o tipo de ponta de pulverização utilizado.

Analisando a tabela 1, pode-se verificar que tanto para os fatores fungicidas e estratos de coleta de cartões não foram observadas diferenças para nenhuma das variáveis relacionadas com a deposição de gotas analisadas. Para os fungicidas utilizados, tanto o tipo de ingrediente ativo como a adição ou não de adjuvante, no caso de azoxistrobina + ciproconazol e azoxistrobina, não influenciaram no padrão de pulverização. A análise dos estratos de coleta dos cartões, que não mostrou diferenças, assegura a adequação da metodologia de disposição das plantas nos vasos e de disposição dos

Tabela 1 - Comparação de médias de variáveis relacionadas com a deposição para categorias de gotas, fungicidas e estratos de coleta de cartões.

\begin{tabular}{|c|c|c|c|c|c|c|c|c|}
\hline \multicolumn{9}{|c|}{ Categoria de gotas } \\
\hline \multirow[b]{2}{*}{ Gotas finas } & \multicolumn{2}{|c|}{ DMV* } & \multicolumn{2}{|c|}{ NGC } & \multicolumn{2}{|c|}{ DMN } & \multicolumn{2}{|c|}{ ARE } \\
\hline & 161,3 & $\mathrm{c}^{* *}$ & 193,3 & a & 102,0 & $\mathrm{~b}$ & 0,99 & ns \\
\hline Gotas médias & 233,6 & $\mathrm{~b}$ & 143,8 & $\mathrm{~b}$ & 128,2 & a & 0,97 & \\
\hline \multirow[t]{2}{*}{ Gotas grossas } & 359,5 & a & 75,7 & $\mathrm{c}$ & 131,2 & a & 0,98 & \\
\hline & \multicolumn{2}{|c|}{ DMV } & \multicolumn{2}{|c|}{ NGC } & \multicolumn{2}{|c|}{ DMN } & \multicolumn{2}{|c|}{ ARE } \\
\hline Azoxistrobina + ciproconazol + óleo mineral & 253,1 & ns & 134,2 & ns & 122,1 & ns & 0,98 & ns \\
\hline Azoxistrobina + óleo mineral & 249,6 & & 138,1 & & 121,8 & & 0,98 & \\
\hline \multirow[t]{2}{*}{ Ciproconazol } & 251,7 & & 140,6 & & 117,5 & & 0,98 & \\
\hline & \multicolumn{2}{|c|}{ DMV } & \multicolumn{2}{|c|}{ NGC } & \multicolumn{2}{|c|}{ DMN } & \multicolumn{2}{|c|}{ ARE } \\
\hline Superior & 250,7 & ns & 135,4 & ns & 120,8 & ns & 0,97 & ns \\
\hline Médio & 250,3 & & 139,9 & & 118,8 & & 0,98 & \\
\hline Inferior & 253,4 & & 137,5 & & 121,7 & & 0,99 & \\
\hline
\end{tabular}

*DMV: diâmetro mediano volumétrico $(\mu \mathrm{m})$; NGC: número de gotas por $\mathrm{cm}^{2}$; DMN: diâmetro mediano numérico ( $\left.\mu \mathrm{m}\right)$; ARE: amplitude relativa; **Médias seguidas pela mesma letra minúscula nas colunas não diferem estatisticamente entre si pelo teste de Tukey em nível de $5 \%$ de probabilidade de erro; ${ }^{\text {ns }}$ não significativo $(\mathrm{P} \geq 0,05)$.

Ciência Rural, v.41, n.10, out, 2011. 
vasos no terreno no momento da aplicação dos tratamentos, desse modo não influenciando na cobertura dos trifólios e isolando somente o fator da idade dos tecidos das demais variáveis.

Para o número de dias entre a aplicação dos fungicidas e o aparecimento da primeira pústula, a análise de variância dos dados revelou interação de quarto nível entre os fatores considerados, a $1 \%$ de significância. Na tabela 2, são apresentadas as respostas da interação do número de dias para o aparecimento da primeira pústula de $\boldsymbol{P}$. pachyrhizi com relação à idade fisiológica dos trifólios, intervalos de simulação de chuva após a aplicação e fungicidas para o espectro de gotas grossas. Inicialmente, pode-se perceber que a avaliação das médias dos trifólios mostrou diferença de cerca de dois e quatro dias a mais de residual para o terceiro e quinto trifólios, quando comparados ao trifólio mais velho, o que corresponde a uma diferença da ordem de 16 e $32 \%$ de incremento de residual, respectivamente.

Tanto para as diferentes idades dos trifólios como para o efeito da simulação de chuva, o maior residual de controle foi obtido com a aplicação da mistura de azoxistrobina + ciproconazol, seguidos da aplicação de azoxistrobina e ciproconazol, individualmente. A velocidade com que os fungicidas sistêmicos penetram nos tecidos e translocam dentro deles deve-se às suas propriedades físico-químicas. Essas propriedades são mensuradas através de um coeficiente denominado coeficiente de partição ou valor Log P (AZEVEDO, 2007). Ambos os ingredientes ativos utilizados apresentam coeficiente de partição inferior a 3,2, o que determina sua alta velocidade de absorção. A azoxistrobina mostra um valor de 2,5, enquanto que ciproconazol apresenta um valor de 2,9 (AZEVEDO, 2007).

Como esperado, a simples simulação de chuva nas testemunhas de cada nível dos fatores não apresentou influência no desenvolvimento da doença. Considerando os fungicidas aplicados, os dados demonstram que o efeito negativo da chuva está diretamente ligado ao intervalo entre a aplicação e a ocorrência dela. Quando a simulação de chuva ocorreu aos 60 e 120 minutos após a aplicação dos fungicidas,

Tabela 2 - Número de dias entre a pulverização dos fungicidas e o aparecimento da primeira pústula de $\boldsymbol{P}$. pachyrhizi na interação entre idade fisiológica dos trifólios, intervalos de simulação de chuva após a aplicação e fungicidas para a categoria de gotas grossas.

\begin{tabular}{|c|c|c|c|c|c|c|}
\hline \multicolumn{7}{|c|}{ Categoria de gotas grossas } \\
\hline 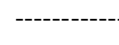 & \multirow[b]{2}{*}{ Test.* } & -- $1^{\circ}$ trifólio** & \multirow{2}{*}{ azox. } & 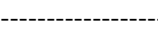 & & \\
\hline & & azox. + cipr. & & cipr. & \multicolumn{2}{|c|}{ Médias } \\
\hline Test** & $4,13 \mathrm{aC}^{* * *}$ & 18,25 aA & $16,63 \mathrm{aB}$ & 17,13 aAB & & \\
\hline 0 ' & $4,13 \mathrm{aD}$ & $11,50 \mathrm{cA}$ & $9,88 \mathrm{~dB}$ & $8,50 \mathrm{dC}$ & & \\
\hline $30^{\prime}$ & $4,38 \mathrm{aC}$ & 14,25 bA & $12,25 \mathrm{cB}$ & $11,75 \mathrm{cB}$ & 11,64 & c \\
\hline $60^{\prime}$ & $4,38 \mathrm{aC}$ & 17,75 aA & $13,25 \mathrm{cB}$ & $12,63 \mathrm{cB}$ & & \\
\hline $120^{\prime}$ & $4,25 \mathrm{aC}$ & 18,63 aA & $15,00 \quad b B$ & 14,25 bB & & \\
\hline & Test. & azox. + cipr. & azox. & cipr. & & \\
\hline Test & $7,13 \mathrm{aC}$ & $19,63 \mathrm{aA}$ & $18,50 \mathrm{aB}$ & $18,38 \mathrm{aB}$ & & \\
\hline $0^{\prime}$ & $7,25 \quad \mathrm{aD}$ & $13,63 \mathrm{cA}$ & $11,38 \mathrm{eB}$ & $10,50 \mathrm{eC}$ & & \\
\hline $30{ }^{\prime}$ & $6,88 \mathrm{aC}$ & $17,38 \quad \mathrm{bA}$ & $13,13 \mathrm{~dB}$ & $12,38 \mathrm{~dB}$ & 13,52 & $\mathrm{~b}$ \\
\hline 60 & $7,00 \mathrm{aC}$ & 19,00 aA & $14,50 \mathrm{cB}$ & $14,25 \mathrm{cB}$ & & \\
\hline $120^{\prime}$ & $7,13 \mathrm{aC}$ & 19,63 aA & $16,63 \mathrm{bB}$ & 16,13 bB & & \\
\hline & Test. & azox. + cipr. & azox. & cipr. & & \\
\hline Test & $8,63 \mathrm{aC}$ & $20,38 \quad \mathrm{bA}$ & $20,00 \quad \mathrm{aAB}$ & $19,50 \quad \mathrm{aB}$ & & \\
\hline $0^{\prime}$ & $9,00 \quad \mathrm{aD}$ & $15,38 \mathrm{dA}$ & $13,38 \quad \mathrm{~dB}$ & $12,00 \mathrm{eC}$ & & \\
\hline $30^{\prime}$ & $8,63 \mathrm{aD}$ & $19,63 \mathrm{cA}$ & $15,63 \mathrm{cB}$ & $14,50 \mathrm{dC}$ & 15,39 & a \\
\hline $60^{\prime}$ & $8,88 \quad \mathrm{aD}$ & 20,25 bcA & $17,88 \quad b B$ & $16,38 \mathrm{cC}$ & & \\
\hline $120^{\prime}$ & $9,13 \mathrm{aD}$ & $21,50 \mathrm{aA}$ & $19,88 \mathrm{aB}$ & 17,38 bC & & \\
\hline Médias & $6,73 \mathrm{D}$ & $17,78 \quad \mathrm{~A}$ & 15,19 В & $14,38 \quad \mathrm{C}$ & & \\
\hline
\end{tabular}

*Test.: testemunha para fungicidas; azox. + cipr.: azoxistrobina + ciproconazol; azox.: azoxistrobina; cipr.: ciproconazol. **Test.: testemunha para chuva simulada; 0, 30, 60, 120: tempos, em minutos, decorridos entre a aplicação dos tratamentos e a simulação de chuva. ***Médias seguidas pela mesma letra maiúscula nas linhas e minúscula nas colunas não diferem estatisticamente entre si pelo teste de Tukey em nível de $5 \%$ de probabilidade de erro. **** A idade está denominada de primeiro a quinto trifólio, sendo que o primeiro trifólio refere-se ao trifólio mais velho. 
a remoção destes foi menor, sendo que, para muitas das situações, mesmo chuva 60 minutos após a aplicação não apresentou influência sobre a absorção dos produtos. Mesmo a ocorrência de chuva imediatamente após a aplicação dos produtos ( $\left.0^{\prime}\right)$ diferiu da testemunha sem aplicação de fungicida, sinalizando, dessa forma, que parte do ingrediente ativo aplicado é prontamente absorvido pelos tecidos da planta logo após o contato com eles.

Considerando a categoria de gotas médias (Tabela 3), verificou-se aumento significativo do número de dias para o aparecimento da primeira pústula, quando comparada à categoria de gotas grossas. Seguindo a mesma tendência do verificado para a categoria de gotas grossas, as diferenças de residual entre primeiro, terceiro e quinto trifólios foram de $16 \mathrm{e}$ $28 \%$, resultados estes que indicam uma expressiva consistência da resposta baseada na idade dos tecidos. Salienta-se que, mesmo variando o DMV das gotas utilizadas para obtenção de espectro de gotas médias, apesar de ter sido verificada resposta de aumento do número de dias de residual, as diferenças entre os trifólios, em percentagem, mantiveram-se as mesmas.

A análise de comparação entre os fungicidas mostrou resposta superior de residual quandoutilizada a mistura azoxistrobina + ciproconazol. Para a testemunha sem simulação de chuva, verificouse, para o primeiro trifólio, que todos os fungicidas testados apresentaram resposta semelhante entre si, porém, para o terceiro e quinto trifólios, azoxistrobina + ciproconazol foram superiores na manutenção do residual. Fato este também verificado para todos os níveis do fator chuvas em que a mistura dos ingredientes ativos se sobressaiu em comparação com a sua aplicação individual.

Os intervalos entre a aplicação dos tratamentos e a simulação de chuva tiveram o efeito reduzido à medida que o tempo decorrido aumentou. Considerando a mistura dos fungicidas, para todos os trifólios avaliados, mesmo a simulação de chuva aos 60 minutos após a aplicação não apresentou influência sobre o fungicida, sendo que o número de dias para o

Tabela 3 - Número de dias para o aparecimento da primeira pústula de $\boldsymbol{P}$. pachyrhizi na interação entre idade fisiológica dos trifólios, intervalos de simulação de chuva após a aplicação e fungicidas para a categoria de gotas médias.

\begin{tabular}{|c|c|c|c|c|c|}
\hline \multicolumn{6}{|c|}{ Categoria de gotas médias } \\
\hline & \multirow[b]{2}{*}{ Test.* } & - $1^{\circ}$ trifólio*: & ----- & ------------- & \\
\hline & & azox. + cipr. & azox. & cipr. & \multirow[t]{2}{*}{ Médias } \\
\hline Test** & $4,25 \mathrm{aB}^{* * *}$ & 19,38 aA & 18,38 aA & 18,25 aA & \\
\hline 0 ' & $4,25 \mathrm{aC}$ & $15,00 \mathrm{cA}$ & $14,75 \mathrm{cA}$ & $11,50 \quad \mathrm{cB}$ & \\
\hline $30^{\prime}$ & $4,25 \mathrm{aD}$ & 16,88 bA & $14,75 \mathrm{cB}$ & $12,13 \mathrm{cC}$ & $13,21 \quad \mathrm{c}$ \\
\hline $60^{\prime}$ & $4,25 \mathrm{aC}$ & 18,50 aA & $15,88 \mathrm{bcB}$ & $15,50 \quad b B$ & \\
\hline \multirow[t]{2}{*}{120} & $4,25 \mathrm{aC}$ & $19,63 \mathrm{aA}$ & $16,50 \mathrm{bB}$ & $16,00 \quad b B$ & \\
\hline & Test. & azox. + cipr. & azox. & cipr. & \\
\hline Test & $7,25 \mathrm{aC}$ & 21,00 aA & $19,88 \mathrm{aAB}$ & $19,63 \mathrm{aB}$ & \\
\hline 0 ' & $7,00 \mathrm{aD}$ & 17,75 bA & $15,88 \mathrm{cB}$ & $13,25 \mathrm{dC}$ & \\
\hline $30^{\prime}$ & $7,00 \mathrm{aD}$ & 19,88 aA & $17,25 \quad b B$ & $15,13 \mathrm{cC}$ & $15,34 \quad b$ \\
\hline $60^{\prime}$ & $7,00 \quad \mathrm{aC}$ & 20,38 aA & $18,00 \mathrm{bB}$ & 17,13 bB & \\
\hline \multirow[t]{2}{*}{$120^{\prime}$} & $6,75 \mathrm{aC}$ & 20,75 aA & $18,00 \quad b B$ & $18,00 \quad b B$ & \\
\hline & Test. & azox. + cipr. & azox. & cipr. & \\
\hline Test & $8,88 \mathrm{aC}$ & $22,50 \mathrm{aA}$ & 20,75 abB & $20,13 \mathrm{aB}$ & \\
\hline 0 ' & $8,63 \mathrm{aD}$ & $18,50 \mathrm{cA}$ & $17,63 \mathrm{cB}$ & $14,25 \mathrm{eC}$ & \\
\hline $30^{\prime}$ & $9,25 \mathrm{aC}$ & 20,88 bA & $17,63 \mathrm{cB}$ & $17,00 \mathrm{~dB}$ & 16,84 a \\
\hline $60^{\prime}$ & $8,63 \mathrm{aD}$ & $22,63 \mathrm{aA}$ & $21,25 \mathrm{aB}$ & $18,13 \mathrm{cC}$ & \\
\hline $120^{\prime}$ & $9,13 \mathrm{aD}$ & $22,13 \mathrm{aA}$ & 20,13 bB & 18,88 bC & \\
\hline Médias & $6,72 \mathrm{D}$ & $19,72 \mathrm{~A}$ & 17,78 & $16,33 \mathrm{C}$ & \\
\hline
\end{tabular}

*Test.: testemunha para fungicidas; azox. + cipr.: azoxistrobina + ciproconazol; azox.: azoxistrobina; cipr.: ciproconazol. **Test.: testemunha para chuva simulada; 0, 30, 60, 120: tempos, em minutos, decorridos entre a aplicação dos tratamentos e a simulação de chuva. ***Médias seguidas pela mesma letra maiúscula nas linhas e minúscula nas colunas não diferem estatisticamente entre si pelo teste de Tukey em nível de 5\% de probabilidade de erro. $* * * *$ A idade está denominada de primeiro a quinto trifólio, sendo que o primeiro trifólio refere-se ao trifólio mais velho.

Ciência Rural, v.41, n.10, out, 2011. 
aparecimento da primeira pústula foi comparável à testemunha sem aplicação de chuva.

No que se refere à aplicação dos ingredientes ativos individualmente, a reposta foi diferente. Em todas as situações, mesmo na simulação de chuva aos 120 minutos após a aplicação, houve redução na eficiência de manutenção do residual dos ativos.

Quando se considerou a aplicação de gotas de categoria fina (Tabela 4), pode-se perceber mais uma vez a relação de incremento de residual, em função da idade dos trifólios, muito próxima à já evidenciada para os espectros com DMV maior. Para as gotas finas, a diferença verificada foi de 15 e $28 \%$ para o terceiro e quinto trifólios, respectivamente, quando comparados ao primeiro trifólio.

Com a utilização do espectro de gotas finas, o maior número de dias para o aparecimento da primeira pústula foi observado, sendo as médias de 13,85; 15,92 e 17,71 dias para o primeiro, terceiro e quinto trifólios. Para este espectro de gotas, mais uma vez, verificou-se que a mistura dos ingredientes ativos azoxistrobina + ciproconazol proporcionou o maior incremento de residual, tendo uma média de 20,78 dias. Em contrapartida, esse residual foi de 18,57, 17,06 e 6,93 para azoxistrobina, ciproconazol e para a testemunha sem aplicação, respectivamente.

Percebe-se claramente através da análise dos dados, primeiramente comparando a idade dos trifólios, que a percentagem de incremento de residual decresce à medida que se consideram tecidos mais novos na planta. Essa relação é inversamente proporcional à absorção dos fungicidas quando as gotas são de categoria grossa, ou seja, mesmo com a aplicação de gotas de DMV elevado e com menor número de gotas por $\mathrm{cm}^{2}$ tecidos mais novos têm maior capacidade de absorção dos fungicidas. Tal fato é observado tanto para gotas médias quanto para gotas finas.

Quando são comparados os diferentes espectros de gotas dentro de um mesmo trifólio, as diferenças ficam ainda mais evidentes. Sempre que é

Tabela 4 - Número de dias para o aparecimento da primeira pústula de $\boldsymbol{P}$. pachyrhizi na interação entre idade fisiológica dos trifólios, intervalos de simulação de chuva após a aplicação e fungicidas para a categoria de gotas finas.

\begin{tabular}{|c|c|c|c|c|c|c|}
\hline \multicolumn{7}{|c|}{ Categoria de gotas finas } \\
\hline & \multirow[b]{2}{*}{ Test.* } & \multirow{2}{*}{\multicolumn{2}{|c|}{ azox. + cipr. }} & \multirow[b]{2}{*}{ azox. } & \multirow[b]{2}{*}{ cipr. } & \\
\hline & & & & & & \multirow[t]{2}{*}{ Médias } \\
\hline Test** & $4,50 \quad \mathrm{aC}^{* * *}$ & 20,13 & $\mathrm{aA}$ & $18,88 \mathrm{aB}$ & $17,88 \quad \mathrm{aB}$ & \\
\hline 0 ' & $4,25 \mathrm{aD}$ & 15,75 & $\mathrm{cA}$ & $14,63 \mathrm{cB}$ & $13,13 \mathrm{dC}$ & \\
\hline $30^{\prime}$ & $4,50 \mathrm{aD}$ & 17,88 & $\mathrm{bA}$ & $16,63 \mathrm{bB}$ & $12,63 \mathrm{dC}$ & $13,85 \mathrm{c}$ \\
\hline $60^{\prime}$ & $4,38 \mathrm{aC}$ & 20,50 & $\mathrm{aA}$ & $17,63 \mathrm{bB}$ & 16,63 bB & \\
\hline \multirow[t]{2}{*}{$120^{\prime}$} & $4,50 \mathrm{aD}$ & 20,38 & $\mathrm{aA}$ & $17,00 \quad b B$ & $15,38 \mathrm{cC}$ & \\
\hline & Test. & \multicolumn{2}{|c|}{ azox. + cipr. } & azox. & cipr. & \\
\hline Test & $7,38 \mathrm{aD}$ & 22,13 & abA & $20,63 \mathrm{aB}$ & $19,88 \quad \mathrm{aC}$ & \\
\hline $0^{\prime}$ & $7,38 \quad \mathrm{aD}$ & 17,63 & $\mathrm{dA}$ & $16,50 \mathrm{~dB}$ & $15,13 \mathrm{dC}$ & \\
\hline $30^{\prime}$ & $7,50 \mathrm{aD}$ & 20,25 & $\mathrm{cA}$ & $17,63 \mathrm{cB}$ & $15,50 \mathrm{dC}$ & $15,92 \mathrm{~b}$ \\
\hline $60^{\prime}$ & $7,13 \mathrm{aC}$ & 22,38 & $\mathrm{aA}$ & $18,63 \mathrm{bB}$ & 18,13 bB & \\
\hline \multirow[t]{2}{*}{120} & $7,38 \quad \mathrm{aD}$ & 21,75 & bA & 18,25 bB & $17,38 \quad \mathrm{cC}$ & \\
\hline & Test. & \multicolumn{2}{|c|}{ azox. + cipr. } & azox. & cipr. & \\
\hline Test & $9,25 \mathrm{aD}$ & 24,75 & $\mathrm{aA}$ & $23,25 \mathrm{aB}$ & 22,13 aC & \\
\hline $0^{\prime}$ & $8,88 \mathrm{aD}$ & 18,63 & $\mathrm{dA}$ & $17,63 \mathrm{~dB}$ & $16,63 \mathrm{dC}$ & \\
\hline $30^{\prime}$ & $8,63 \mathrm{aD}$ & 22,00 & $\mathrm{cA}$ & $18,75 \mathrm{cB}$ & $17,38 \mathrm{dC}$ & $17,71 \quad \mathrm{a}$ \\
\hline $60^{\prime}$ & $9,25 \mathrm{aD}$ & 23,63 & $\mathrm{bA}$ & 21,00 bB & $18,63 \mathrm{cC}$ & \\
\hline $120^{\prime}$ & $9,13 \mathrm{aD}$ & 23,88 & $\mathrm{bA}$ & 21,50 bB & $19,50 \quad b C$ & \\
\hline Médias & $6,93 \quad \mathrm{D}$ & 20,78 & A & $18,57 \quad \mathrm{~B}$ & $17,06 \quad \mathrm{C}$ & \\
\hline
\end{tabular}

*Test.: Testemunha para fungicidas; azox. + cipr.: azoxistrobina + ciproconazol; azox.: azoxistrobina; cipr.: ciproconazol. **Test.: Testemunha para chuva simulada; 0, 30, 60, 120: tempos, em minutos, decorridos entre a aplicação dos tratamentos e a simulação de chuva. ***Médias seguidas pela mesma letra maiúscula nas linhas e minúscula nas colunas não diferem estatisticamente entre si pelo teste de Tukey em nível de 5\% de probabilidade de erro. **** A idade está denominada de primeiro a quinto trifólio, sendo que o primeiro trifólio refere-se ao trifólio mais velho. 
utilizado um espectro de gotas que proporcione maior número de gotas por $\mathrm{cm}^{2} \mathrm{e}$, consequentemente, maior cobertura da área foliar, observa-se maior absorção e em taxa mais elevada. Apesar de os tecidos mais novos terem mostrado maior taxa de absorção, mesmo com a utilização de gotas grossas, maior é a diferença de incremento de residual com a diminuição do DMV utilizado, o que reforça a hipótese de que tecidos mais novos propiciem absorção mais rápida dos fungicidas, assim que estes são colocados em contato com os tecidos.

\section{CONCLUSÃO}

Gotas de menor diâmetro mediano volumétrico apresentaram maior velocidade de absorção de fungicidas devido ao maior número de gotas por centímetro quadrado e, consequentemente, maior área específica de contato produto/planta. Trifólios mais novos absorvem os fungicidas mais rapidamente e, em consequência, estabelecem um maior atraso no desenvolvimento da doença. A utilização da mistura de azoxistrobina + ciproconazol proporciona maior número de dias até o aparecimento da primeira pústula. A influência da chuva sobre o período de proteção das plantas possui relação altamente dependente do intervalo entre a aplicação dos produtos e a ocorrência daquela, do produto que está sendo aplicado e da idade dos tecidos considerados.

\section{REFERENCIAS}

AZEVEDO, L.A.S. Fungicidas sistêmicos - Teoria e prática. Campinas: EMOPI, 2007. 284p

DEBONA, D. et al. Efeito de níveis de cálcio e sombreamento em plantas de soja sobre a infecção por Phakopsora pachyrhizi. Tropical Plant Pathology, v.33, n.5, p.388-389, 2008. Disponível em: <http://www.scielo.br/scielo.php?pid=S1982$56762008000500009 \&$ script $=$ sci_arttext $>$. Acesso em: 03 set. 2010. doi: 10.1590/S1982-56762008000500009.

DEBORTOLI, M.P. Efeito do "rainfastness" e adjuvante na aplicação de fungicidas foliares em cultivares de soja. 2008. 57f. Dissertação (Mestrado em Engenharia Agrícola) Universidade Federal de Santa Maria, Santa Maria, RS.

FEHR, W.R. et al. Stage of development descriptions for soybeans, Glycine $\max$ (L.) Merrill. Crop Science, v.11, n.6, p.929-931, 1971 .

FURTADO, G.Q. et al. Influência do estádio fenológico e da idade dos trifólios de soja na infecção de Phakopsora pachyrhizi. Tropical Plant Pathology, v.34, n.2, p.118-122. 2009. Disponível em: $<$ http://www.scielo.br/scielo.php? script=sci_arttext\&pid=S1982$56762009000200007 \& \operatorname{lng}=\mathrm{pt} \& \mathrm{nrm}=\mathrm{iso} \& \ln \mathrm{g}=\mathrm{pt}>$. Acesso em: 03 set. 2010. doi: 10.1590/S1982-56762009000200007.

OLIVEIRA, S.H.F. et al. Efeito da chuva sobre a tenacidade e eficiência de fungicidas cúpricos associados ao óleo vegetal no controle da ferrugem do cafeeiro. Fitopatologia Brasileira, v.27, n.6, p.581-585, 2002. Disponível em: <http:// www.apsnet.org/publications/PlantDisease/BackIssues/ Documents/1994Articles/PlantDisease78n09_857.pdf>. Acesso em: 03 set. 2010 . doi: 10.1590/S0100-41582002000600004.

SILVA, F.A.S.; AZEVEDO, C.A.V. Versão do programa computacional Assistat para o sistema operacional Windows. Revista Brasileira de Produtos Agroindustriais, v.4, n.1, p.71-78, 2002. 